\title{
Determination of the characteristics of ocean inhomogeneities by the times structure of an acoustic signal
}

\author{
V.N. FOKIN, A.G. NECHAEV and M.S. FOKINA
}

Institute of Applied Physics, Russian Academy of Sciences, 46 Uljanov St., 603600 Nizhny Novgorod, Russia

\begin{abstract}
In this report the method of ray differential acoustic tomography of the ocean is discussed. The method is based on the fact that the mean velocity of sound pulse propagation along the ray trajectory depends on the angle of the ray exit. The method efficiency is analyzed numerically for the signal reception to the horizontal array and the single hydrophone. It is shown that the signal of illumination may be suppressed and the scattered signal may be separated by time gating: It is also shown that the depth of the deposition of a space-localized inhomogeneity may be estimated by the width of the scattered signal autocorrelation function. The distance to the inhomogeneity may be defined by the cross correlation function of the scattered signal at various array receivers. The influence of the depth of the submergence of a scatterer and the distance between a source and a receiver on the scattered signal structure is analyzed.
\end{abstract}

\section{INTRODUCTION}

Various aspects of the space-time structure of the "illumination" field were repeatedly discussed in literature (see, e.g., [1,2]), whereas problems on mutual relation of time delay values of the signals propagating along the path "source-scattering inhomogeneity-receiver" and on the inhomogeneity position were not considered for long acoustic paths, though a similar question for very short paths was dealt with in [3].

Here we discussed the essential element of differential method - spatial localization of ocean inhomogeneities characteristics. We assume that the source radiates short sound pulse. Then the main contribution into the scattered field intensity make only that elements of scattered volume for which $\left|\tau-t_{s}-t_{\tau}\right| \leq \tau_{0} / r$ and which are the region of intersection of ray paths transmitted from the source and the receiver. Where $\tau_{0}-$ sound pulse duration after consistent processing, $t_{s}-$ time delay values of the signals propagating along the path "source-scattering inhomogeneity-receiver", $t_{\tau}-$ time delay values of the signals propagating along the path "source-receiver". These two conditions permits us to localize the scatterers.

\section{NUMERICAL SIMULATION RESULTS}

In this report space-time structure of the "scattered" field intensity were numerically investigated. For bulk inhomogeneities below we shall consider the dependences $T(x)$ and $T(z)$, where $T$ is the total time of the scattered signal travel along the path "source-scatterer-receiver", $x_{z}$ is the distance from the source to the scatterer, $z$ is the depth of the scatterer location. 
We also made an assumption on point scatterers, which permitted us to model the "illumination", the scattered signal and their sum. It has been assumed that the signal received by a horizontal 32element array oriented along the acoustic path, while the radiated signal has the form $p_{0}(0, t)=\delta(t)$, where $\delta(t)$ is the delta function. The distance between the receiving elements of the array was equal in all cases to $250 \mathrm{~m}$. The computation were performed using the program based on the ray one [6]. Since the main goal was to obtain a space-time pattern, it was assumed in the calculations that the bottom is an absolutely reflecting one, whereas the scattering cross-section (or the bulk scattering coefficient) equals 1 for all incidence and scattering angles of plane waves.

Let us consider first a relatively short path of length up to $100 \mathrm{~km}$ using the example of a waveguide with a constant gradient of the sound speed $d c / d z=1.67 * 10^{-2} 1 / c$, and the depth $5 \mathrm{~km}$ (the results obtained remain qualitatively valid for a wide class of underwater surface waveguides). The structure of the signal scattered by a point scatterer for 9 discrete distances to the scatterer is shown in Fig.1. The plots are arranged close to each other for the sake of illustrativity. The upper plot is the time structure of the "illumination" signal. The time delay is plotted on the horizontal axis, while the signal amplitude in the logarithmic scale is plotted on the vertical one. The plots corresponding to the array elements which are most remote from the source, are displaced vertically downwards with respect to the plot of the first receiver. To compensate the time delay occurred when a signal travels the distance between the array elements, the signal of all receivers were shifted along the time axis by the value $\left(-D_{n} / c\right)$, where $D_{n}$ is the distance from the $n$-th array element to the element nearest to the source. The distance from the source to the first receiver was equal to $50 \mathrm{~km}$, the submersion depth of the source is $100 \mathrm{~m}$, of the receivers $150 \mathrm{~m}$ and of the scatterer $50 \mathrm{~m}$.

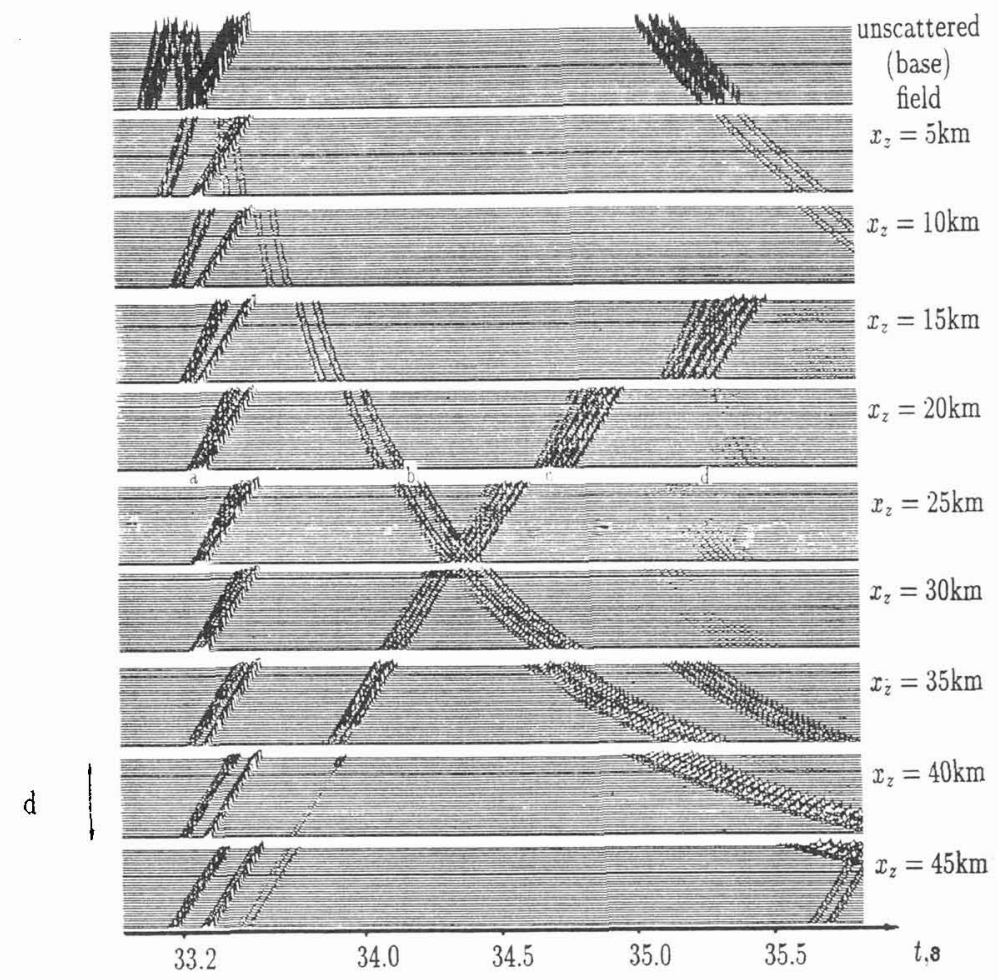

Fig. 1 The structure of the signal scattered by a point scatterer for 9 discrete distances to the scatterer $(b-j)$ and received by a horizontal 32 -element array. The upper plot is the time structure of the "illumination" signal. 
Four main lines $A, B, C$ and $D$ are well seen in Fig. 1. The doublet of lines $\mathrm{A}$ is formed by the signal corresponding to the case of scattered water rays which came out of the source into water rays (water-water), the line $B$ corresponds to the scattering of bottom rays transmitted from the source and singly reflected from the bottom (bottom-water), the line $C$ is associated with the scattering of water rays emitted from the source into the rays singly reflected from the bottom after scattering (water-bottom) and the line $D$ corresponds to the scattering of bottom rays into bottom ones (bottom-bottom). As is seen, the lines A and $D$ are completely masked by the "illumination" signal, while the lines $B$ and $C$ lie outside the time interval occupied by the "illumination". Therefore, suppression of the "illumination" consists in singling out the time interval between the A and $D$ lines.

After the "illumination" signal is suppressed, inhomogeneities are being localized in each element of the receiving array by means of time gating of the scattered pulse. The position of the time gate is determined by the assumed location of the scatterer on the path and by the $B$ and $C$ lines, along which the scattered signal is localized. However, in this case (Fig.1) there is some ambiguity in the inhomogeneity localization, since two positions of the scatterer are possible for each time gate (except for the gate which distinguishes the region of the $B$ and $C$ intersection). This ambiguity can be easily eliminated, e.g., by submerging the receivers. If the receivers are placed near the bottom, only the $C$ and $D$ lines remain in the plot, while the $A$ and $B$ lines vanish. The ambiguity can be eliminated without changing the geometry of the experiment. In this case the position of the time gate must vary from one receiver to another in the array according to the lines $B$ and $C$. The ambiguity can be removed due to opposite signs of the derivative lines $B$ and $C$ along the array elements. The position of the lines $B$ and $C$ can either be preliminarily calculated or obtained by means of previous calibration of the waveguide using an echo sensor. By exhaustion of gates corresponding to various distances to inhomogeneities, we can judge the inhomogeneity position by the maximum response of the array.

To investigate the dependences of scattered signal structure on the depth of inhomogeneity occurrence, it proves to be enough to restrict ourselves to the consideration of the dependences $T(x)$ in a single receiver, where $x$ is the distance to the inhomogeneity, since the dependence $T(x)$ is analogous to the scattered signal structure of the array.

On the assumption that the scatterers are distributed over the entire depth of the waveguide from the source to the receiver, the scattered signal does not have a distinct structure and occupies the whole time interval beginning with the travel times of fastest water rays up to the latest received bottom reflections. Figure 2a (the calculation conditions correspond to those Fig.1) displays the dependence $T(x)$ for the scatterers covering the whole bulk of the waveguide.

The time structure is essentially simplified if the scatterers are localized within a relatively small depth interval. Figures $2 b$ and $2 c$ illustrate the cases when the scatterers are located in the layers $0-100 m$ and $400-500 m$ (the rest calculation conditions are the same as in Fig.1). It is evident that the lines in Fig. $2 \mathrm{~b}$ as well as the lines in Fig.1 can be classified as follows. The doublet of the lines I and II corresponds to the line $A$ in Fig.1, the line III to the line $B$, IV to $C$ and $V$ to $D$. The line VI is produced by scattering of rays having a single reflection from the bottom to the scatterer, into the rays doubly reflected from the bottom after it, while the line VII is due to scattering of rays doubly interacting with bottom before the scatterer, into the rays singly interacting with the bottom after it.

A comparison of Fig. $2 b$ and $2 c$ shows variation of the scattered signal structure with increasing depth of the scatterer layer occurrence. The general character of the dependence $T(x)$ is conserved, though every line is splitted. The splitting value is proportional to the depth of the scatterer localization and is related to the time delay between the rays which reach the receiver with reflection from the surface and without it. The latter circumstance can be used in schemes of signal processing. The search over the depth will be reduced to the determination of the time autocorrelation function maximum of a signal in an individual receiver.

Note that the structure $T(x)$ does not depend on the distance between the source and the receiver, since if the scatterers are located within the depth interval $400-500 \mathrm{~m}$ and an acoustic path length is 
$100 \mathrm{~km}$, the dependence $T(x)$ (Fig.2d) is analogous to Fig.2b. The relative delays of signals diminish, while the absolute ones, naturally, increase.

The unambiguous coupling of the $T(x)$ dependence branches with incidence angles of scattered signals can be employed to localize the scatterers. For example, when receiving signals in the range of angles which correspond to purely water rays from the "illumination" signal, only water signals remain, while in the scattered signal in Fig.2b, the branches I, II, III are conserved.

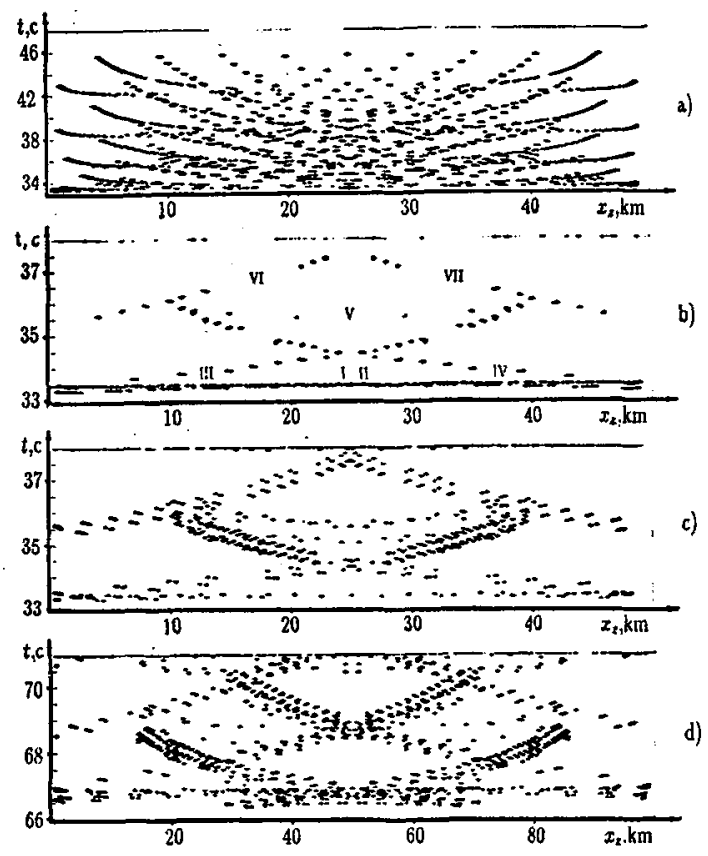

Fig. 2 The time structure of the scattered signal ( $T$ is the time, $x_{z}$ is the distance to the scattering inhomogeneities) for the different depths of inhomogeneities localization and different distances between source and receiver.

\section{CONCLUSIONS}

Therefore, application of pulse signals, the duration of which after matched filtration is shorter than the intervals between separate arrivals of "illumination" signals, and of time gating enables us to single out the scattered signals. Further analysis of time structure of the scattered field allows for obtaining information on space distribution of ocean inhomogeneity characteristics.

\section{References}

[1] Galkin O.P., Ocean Acoustics ( Nauka, M., 1982) pp.92-106.

[2] Galkin O.P., Shvachko L.V., Kharchenko E.A. et. al., Problem of Ocean Acoustics ( Nauka, M., 1984) pp.118-133.

[3] Gostev V.S. and Shvachko R.F., Dokl. Akad. Nauk SSSR 282 (1985) 1082-1085.

[4] Fokin V.N. and Fokina M.S., Akust. Zh. 37 (1991) 782-788. 